\title{
Ascitic fluid $\gamma$ interferon concentrations and adenosine deaminase activity in tuberculous peritonitis
}

\author{
M A Sathar, A E Simjee, Y M Coovadia, P N Soni, S A H Moola, B Insam, F Makumbi
}

\begin{abstract}
The $\gamma$ interferon $(\gamma$-IFN) concentration and the adenosine deaminase (ADA) activity were evaluated in 30 patients with tuberculous peritonitis, 21 patients with ascites due to a malignant disorder, and 41 patients with cirrhosis. The $\gamma$-IFN concentrations were significantly higher $(p<0.0001)$ in tuberculous peritonitis patients (mean: $6 \cdot 70 \mathrm{U} / \mathrm{ml}$ ) than in the malignant (mean: $3.10 \mathrm{U} / \mathrm{ml}$ ) and cirrhotic (mean: 3.08 $\mathrm{U} / \mathrm{ml}$ ) groups. Use of a cut off value of $\geqslant 3 \cdot 2 \mathrm{U} / \mathrm{ml}$ gave the assay a sensitivity of $93 \%$ (25 of 27), a specificity of $98 \%$ (54 of 55), positive ( $P+$ ) and negative $(P-)$ predictive values of $96 \%$ and a test accuracy of $96 \%$. The ADA activity was significantly $(p<0.0001)$ higher in the tuberculous peritonitis group (mean: $101.84 \mathrm{U} / \mathrm{l})$ than in the control groups (cirrhosis (mean: 13.49 U/1) and malignancy (mean: 19.35 U/I)). A cut off value of $>30$ U/I gave the ADA test a sensitivity of $93 \%$ (26 of 28 ) a specificity of $96 \%$ (51 of $53)$, a $(\mathbf{P}+)$ value of $93 \%$, a $(\mathbf{P}-)$ value of $96 \%$, and a test accuracy of $95 \%$. There was a significant $(p<0.0001)$ correlation $(r=0.72)$ between ADA activity and $\gamma$-IFN values in patients with tuberculous peritonitis. These results show that a high concentration of $\gamma$-IFN in ascitic fluid is as valuable as the ADA activity in the diagnosis of tuberculous peritonitis. Both are rapid non-invasive diagnostic tests for tuberculous peritonitis.
\end{abstract}

(Gut 1995; 36: 419-421)

Keywords: $\gamma$ interferon, adenosine deaminase activity, tuberculous peritonitis.

Gastrointestinal Unit, Departments of Medicine and Medical
Mepartments of Medicine and University of Natal, King Edward VIII Hospital, Durban, South Africa M A Sathar A E Simjee Y M Coovadia P N Soni S A H Moola B Insam

F Makumbi

Correspondence to Mr M A Sathar, Gastrointestinal Unit, PO Gox 17039, Congella 4013, Box 17 .

Accepted for publication 24 June 1994
Tuberculous peritonitis is an endemic disease among socioeconomically disadvantaged communities in both developing and developed countries. ${ }^{1-6}$ With Mycobacterium tuberculosis now emerging as a major pathogen in HIV positive patients, the impact of AIDS and HIV infection on tuberculosis (TB) is of great concern, especially in the heterosexual black population of South Africa in whom TB is endemic and AIDS is an increasingly growing problem. ${ }^{7}$ This is likely to have significant implications for the diagnosis and management of TB in this population group. The diagnosis of abdominal TB is made difficult because of the paucity of $M$ tuberculosis in peritoneal fluid. ${ }^{5}$ Therefore, new techniques which are rapid and less invasive, but which maintain high sensitivities, specificities, and positive/negative predictive values need to be evaluated to aid in the diagnosis of tuberculous peritonitis. A recent major advance in the diagnosis of tuberculous peritonitis is the determination of adenosine deaminase (ADA) activity in the ascitic fluid..$^{8-13} \gamma$ interferon $(\gamma$-IFN), secreted by antigen-triggered CD4+ lymphocytes, is a key lymphokine that activates macrophages, increasing their bactericidal activity against $M$ tuberculosis. ${ }^{14}$ High $\gamma$-IFN concentrations have been reported in patients with tuberculous pleural effusion ${ }^{15}$ and tuberculous peritonitis. ${ }^{16}$ We prospectively measured the $\gamma$-IFN concentrations in patients with tuberculous peritonitis as an alternative rapid and non-invasive test for diagnosing tuberculous peritonitis.

\section{Patients and methods}

Peritoneoscopy was performed on 92 consecutive patients with ascites. There were three patient groups; 30 patients with tuberculous peritonitis ( 29 blacks and 1 Indian; age range: 13-69 years, mean: 37 years); 21 patients with ascites due to malignant disorders (20 blacks and 1 Indian; age range $24-78$ years, mean: 52 years), and 41 blacks with cirrhosis (age range: 19-76 years, mean: 47 years). Routine peritoneal withdrawal of $50 \mathrm{ml}$ of ascitic fluid from all patients was used to culture $M$ tuberculosis on Lowenstein-Jensen media. Ascitic fluid ADA activity was determined spectrophotometrically. ${ }^{17} \mathrm{~A}$ portion of the sedimented deposit of an additional one litre of ascitic fluid obtained from all patients was used to culture the tubercle bacillus. The remainder of the sediment was resuspended in $5 \mathrm{ml}$ of sterile phosphate buffered saline (PBS), $\mathrm{pH} 7 \cdot 2$ and assayed for ascitic $\gamma$-IFN concentrations using a commercially available radioimmunoassay (RIA) kit (IMRX Interferon-gamma RIA, Centocor, Malvern, PA).

Statistical analysis was done using the Statistical Analysis System (SAS), release 6.03 edition, 1988 (SAS Institute Inc, Cary NC, USA). One way, non-parametric analysis of variance (Kruskal-Wallis) was used to determine differences between patients with TB and control groups. The degree of association between quantitative parameters was calculated by the Spearman coefficient correlation.

\section{Results}

Tuberculous peritonitis was diagnosed in 30 patients. The tubercle bacillus was cultured 
Test parameters of the $\gamma$ interferon $(\gamma-I F N$ and the adenosine deaminase (ADA) tests

\begin{tabular}{lll}
\hline Test parameter & $A D A>30 U /$ & $\gamma-I F N \geqslant 3 \cdot 2 U / m l$ \\
\hline Sensitivity (\%) & $26 / 29(93)$ & $25 / 27(93)$ \\
Specificity (\%) & $51 / 53(96)$ & $54 / 55(98)$ \\
P(+) value (\%) & 93 & 96 \\
P(-) value (\%) & 96 & 96 \\
Accuracy (\%) & 95 & 96 \\
\hline P=predictive &
\end{tabular}

from the sedimented deposit of one litre of ascitic fluid in 14 of $27(52 \%)$ patients. In three patients culture was not done. Acid fast bacillus (AFB) was seen on histology (Ziehl-Neelsen Stain) of the peritoneal biopsy specimen in 19 of $30(63 \%)$ patients, while histology and culture were positive in eight of $30(26 \%)$ patients. In five patients in whom culture and histology were negative for AFB, caseating granulomas were seen at histology of the peritoneal biopsy tissue. Biopsy specimens obtained from the peritoneum showed granulomas in 27 of $30(90 \%)$ TB patients. The sedimented deposit of one litre of ascitic fluid of all 92 patients was used to culture Mycobacterium tuberculosis. The bacterium was not cultured from routine peritoneal taps of $50 \mathrm{ml}$ of ascitic fluid obtained from all patients. Microscopy for AFB was negative in all patients. Two patients were also seropositive for HIV. The diagnosis in the cirrhotic and malignancy groups were confirmed by peritoneoscopy and histology.

The sensitivities, specificities, positive $(\mathrm{P}+)$ and negative $(\mathrm{P}-)$ predictive values, and the overall diagnostic accuracy figures at the different cut off values for ADA and $\gamma$-IFN are shown in the Table.

The ADA activity was determined in 81 and 92 patients. Individual ascitic ADA values in the different groups are shown in Figure 1A. ADA activity was significantly higher in the TB group (range: $0 \cdot 8-318.50 \mathrm{U} / 1$; mean: 101.84 $\mathrm{U} / \mathrm{l}$ ) than in the control groups (cirrhosis (range: 4.1-29.3 U/l; mean: $13.49 \mathrm{U} / \mathrm{l}$ ) and malignancies (range: 5.3-70.02 $\mathrm{U} / \mathrm{l}$; mean:

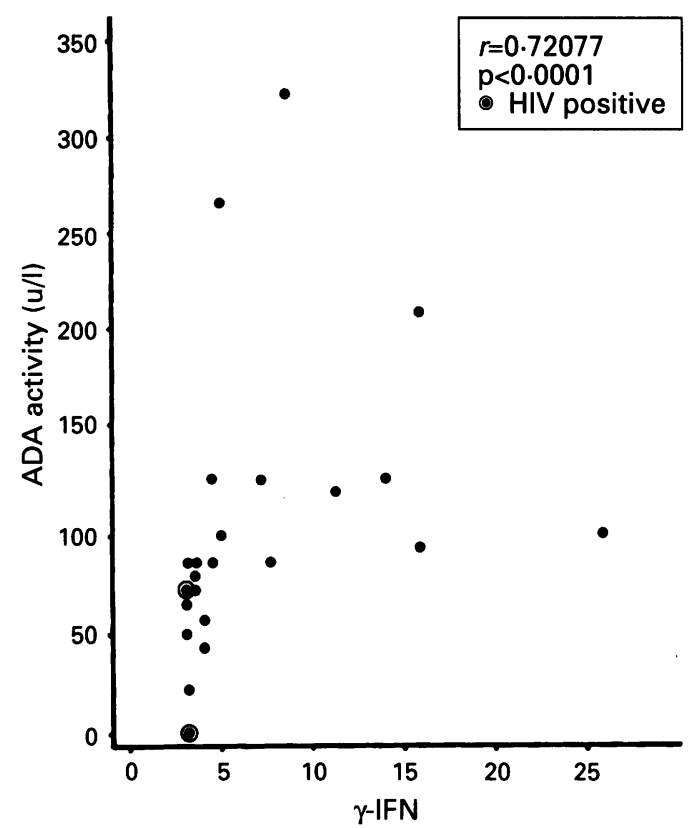

Figure 2: Correlation between ADA activity and $\gamma-I F N$ values in patients with tuberculous peritonitis.

$19.35 \mathrm{U} / \mathrm{l}))(\mathrm{p}<0.0001)$. There was no significant difference between the cirrhotic and malignant groups $(\mathrm{p}<0 \cdot 1099)$.

The $\gamma$-IFN concentrations were determined in 82 of 92 patients. Individual ascitic $\gamma$-IFN values in the different groups are shown in Figure 1B. The cirrhotic (range: $3 \cdot 014-3 \cdot 184$ $\mathrm{U} / \mathrm{ml}$; mean: $3.087 \mathrm{U} / \mathrm{ml}$ ) and malignant (range: $3 \cdot 053-3 \cdot 363 \mathrm{U} / \mathrm{ml}$; mean: $3 \cdot 104 \mathrm{U} / \mathrm{ml}$ ) groups had significantly $(p<0.0001)$ lower $\gamma$-IFN values than the TB group (range: 3.079-25.704 U/ml; mean $6.70 \mathrm{U} / \mathrm{ml})$. The difference in the $\gamma$-IFN concentrations between the cirrhotic and the malignancy groups was not significant $(p<0.6564)$ and the two were combined to form the control group (mean: 3.0955+0.052 U/ml).

There was a significant $(\mathrm{p}<0.0001)$ correlation $(r=0.72)$ between ADA activity and $\gamma$-IFN values in the tuberculous peritonitis group only (Figure 2).

\section{Discussion}

The characteristics of tuberculous peritonitis have been well documented in South Africa. ${ }^{1218}$ The technique of peritoneoscopy is well established and it is used as the gold standard in diagnosing tuberculous peritonitis. Peritoneoscopy and peritoneal biopsies will allow a presumptive diagnosis of TB in $90 \%$ of cases. In areas in which tuberculous peritonitis is endemic, ${ }^{1-4} 19$ including our own, ${ }^{2}$ peritoneoscopy is more useful than either culture $(52 \%)$ or histology $(63 \%)$ in diagnosing tuberculous peritonitis. However, the technique requires trained staff, is invasive, and is not without risk. ${ }^{2} 18$

In most studies, the frequency of a positive culture for $M$ tuberculosis from small volumes of ascitic fluid has been less than $20 \% .^{5}$ In this study, the bacterium was not cultured from $50 \mathrm{ml}$ of ascitic fluid obtained from all TB patients. Menzies et al ${ }^{19}$ reported a $66 \%$ 
success rate (these authors did not indicate the volume of ascitic fluid used). When larger volumes of ascitic fluid (one litre concentrated either by centrifugation or sedimentation) were used, improved culture rates of $63 \%$ (five of eight) ${ }^{18}$ and $83 \%$ (39 of 47$)^{20}$ have been reported. Concentrating one litre of ascitic fluid by sedimentation improved the culture rate to $52 \%$ (14 of 27 ) in this study. Irrespective of the volume of ascitic fluid used, culture of ascitic fluid takes three to eight weeks, which limits its diagnostic usefulness. Thus, some other investigation is almost always necessary. The sensitivity $(93 \%)$ and specificity $(96 \%)$ of the ADA activity in our study confirms our previous observations ${ }^{13}$ and those made by other researchers. ${ }^{8-12} 16$ There were two false negative ADA results. One was a patient with HIV and TB and the other a patient whose diagnosis was established only on the basis of a positive culture of $M$ tuberculosis.

ADA activity has been reported to be significantly lower in patients with AIDS related tuberculous peritonitis ${ }^{16}$ and in those patients in whom the ascitic protein concentration is low. ${ }^{8}$ The latter patient had a total ascitic protein concentration of $42 \mathrm{~g} / 1$ (normal: $60-80$ $\mathrm{g} / \mathrm{l})$. The two false positive $\mathrm{ADA}$ results occurred in patients with ascites due to malignancies, an observation that has been reported by others. ${ }^{11} 16$

Ribera et al ${ }^{16}$ recently reported high $\gamma$-IFN concentrations in 16 patients with tuberculous peritonitis. In their hands the test had a sensitivity and specificity of $100 \%$. The sensitivity and specificity of the $\gamma$-IFN test in our study may be influenced by the fact that tuberculous peritonitis is endemic in our environment ${ }^{2}$ and, perhaps, the manner in which the sample was prepared for the assay. Unlike Ribera et $a l,{ }^{16}$ we observed a significant correlation between the ADA activity and $\gamma$-IFN values in patients with tuberculous peritonitis.

The $\gamma$-IFN values in the two TB patients who were also seropositive for HIV $(3.3 \mathrm{U} / \mathrm{ml})$, were closer to the cut off level, but high enough to differentiate between TB and non-TB. The ADA activity was $<30 \mathrm{U} / 1$ in only one HIV positive patient $(0.8 \mathrm{U} / \mathrm{l})$. Ribera et al ${ }^{16}$ made similar observations in four patients who were HIV positive. $\gamma$-IFN was negative in one of two patients with ascites secondary to a malignancy in whom the ADA activity was falsely positive. Ribera et al ${ }^{14}$ made similar observations in patients with tuberculous pleural effusions secondary to lymphomas. TB is one of the AIDS related opportunistic infections that responds well to treatment, therefore early diagnosis is imperative especially in high risk groups and in areas endemic for $\mathrm{TB}$ and AIDS. ${ }^{7}$ Under such circumstances the $\gamma$-IFN values may prove valuable.

This study shows the significance and value of ascitic $\gamma$-IFN as a definitive diagnostic test for tuberculous peritonitis and also reaffirms the diagnostic value of the ascitic ADA with its high sensitivity and specificity. Both the ADA and the $\gamma$-IFN are simple and rapid non-invasive tests. However, the $\gamma$-IFN test is twice as expensive as the ADA test ( $\$ 10 v \$ 5)$. With greater experience, both tests could potentially supersede invasive procedures for the diagnosis of tuberculous peritonitis, even in developed countries. The value of the ADA and $\gamma$-IFN in AIDS patients with tuberculous peritonitis needs further evaluation.

This work was financed by the University of Natal Research Fund and the South African Medical Research Council (SAMRC). We wish to acknowledge the assistance of Professo Berger of the Department of Chemical Pathology, Mr Hank Breukink of BYK Gulden (SA), Ms Elenor Gouwns of the MRC for statistical analysis of the data and the Medical Superintendent of King Edward VIII Hospital for permission to publish.

1 Lingenfelser T, Zak J, Marks IN, Steyn E, Halkett J, Price SK. Abdominal tuberculosis: Still a potentially lethal disease. Am 7 Gastroenterol 1993; 88: 744-50.

2 Manohar A, Simjee AE, Haffejee AA, Pettengell KE Symptoms and investigative findings in 145 patients with tuberculous peritonitis diagnosed by peritoneoscopy and biopsy over a five year period. Gut 1991; 31: 1130-2.

3 Bhargava DK, Shriniwas, Chopra P, Nijhawan S, Dasarathy S, Kushwaha AKS. Peritoneal tuberculosis: Laparoscopic patterns and its diagnostic accuracy. Am $\mathcal{f}$ Gastroenterol patterns and its diag

4 Al-Quorain AA, Facharzt, Satti MB, Al-Freihi HM, AlGindan YM, Al-Awad N. Abdominal tuberculosis in Saudi Arabia: A clinopathological study of 65 cases. $A m \mathcal{F}$ Gastroenterol 1993; 88: 75-9.

5 Marshall JB. Tuberculosis of the gastrointestinal tract and peritoneum. Am f Gastroenterol 1993; 88: 989-99.

6 Probert CSJ, Jayanti V, Wicks AC, Carr-Locke P, Garner P, Mayberry JF. Epidemiological study of abdominal tuberculosis among Indian migrants and the indigenous population of Leicester, 1972-1989. Gut 1992; 33: 1085-8.

7 Martin DJ, Schoub BD, Miller GB, Sim JGM. AIDS and tuberculosis. S Afr Med 7 1990; 78: 533-5.

8 Fernandez-Rodriguez CM, Perez-Arguelles BS, Ledo L Garcia-Villa LM, Pereira S, Rodriguez-Martinez D. Ascites adenosine deaminase activity is decreased in tuberculous ascites with low protein content. $A m \mathcal{F}$ Gastroenterol 1991; 86: 1500-3.

9 Bhargava DK, Gupta M, Nijhawan S, Dasarathy S. Kushwaha AKS. Adenosine deaminase (ADA) in peritoneal tuberculosis: diagnostic value in ascitic fluid and serum. Tubercle 1990; 71: 121-6.

10 Dwivedi M, Misra SP, Misra V, Kumar R. Value of adenosine deaminase estimation in the diagnosis of tuberculosis sine deaminase estimation in the diagnosis of

11 Voigt MD, Trey C, Lombard C, Kalvaria I, Berman P Kirsch RE. Diagnostic value of ascites adenosine deaminase in tuberculous peritonitis. Lancet 1989; i: 751-4.

12 Martinez-Vazquez JM, Ocana I, Ribera E, Segura RM, Pascual C. Adenosine deaminase activity in the diagnosis of tuberculous peritonitis. Gut 1986; 27: 1049-53.

13 Moodley D, Moola SAH, Simjee AE, Berger GM. diagnostic tests in tuberculous ascites. $S$ Afr Med $\mathcal{f} 1992 ; 81: 108$

14 Ribera E, Espanol T, Martinez-Vazquez JM, Ocana I, Encabo G. Lymphocyte proliferation and gamma interferon production after 'in vivo' stimulation with PPD. Differences between tuberculosis and non-tuberculosis pleurisy in patients with positive tuberculin skin tests. pleurisy in patients with

15 Ribera E, Ocana I, Martinez-Vazquez JM, Rossell M, Espanol T, Ruibal A. High level of interferon gamma in tuberculous pleural effusion. Chest 1988; 93: 308-11.

16 Ribera E, Martinez-Vasquez JM, Ocana I, Ruiz I, Jiminez JG, Encabo G, et al. Diagnostic value of ascites gamma interferon levels in tuberculous peritonitis. Comparison with adenosine deaminase activity. Tubercle 1991; 72: 193-7.

17 Blake J, Berman P. The use of adenosine deaminase assays in the diagnosis of tuberculosis. $S$ Afr Med $f$ 1982; 62 19-21.

18 Geake TMS, Spitaels JM, Moshal MG, Simjee AE Peritoneoscopy in the diagnosis of tuberculous peritonitis. Gastrointestinal Endosc 1981; 27: 66-8.

19 Menzies RI, Fitzgerald JM, Mulpeter K. Laproscopic diagnosis of ascites in Lesotho. BMF 1985; 91: 473-5.

20 Singh MM, Bhargava AN, Jain KP. Tuberculous peritonitis: an evaluation of pathogenic mechanisms, diagnostic procedures and therapeutic measures. $N$ Engl f Med 1969; 281: 1091-4. 